\title{
Tratamento Cirúrgico das Epilepsias
}

\author{
Aziz Rassi Neto* \\ Ricardo Centeno** \\ Andréa V. Amantéa*** \\ Délrio F. da Silva**** \\ Mauro Muszkat****** \\ Maristela Costa****** \\ Sueli Rizzutti******* \\ Ana Regina dos Santos******** \\ Regina S. Alves de Lima*********
}

\begin{abstract}
RESUMO
Pacientes com epilepsia refratária a tratamento clínico podem favorecer-se de uma cirurgia para remoção do foco epileptiforme cortical ou para interrupção desse foco. Antes da cirurgia, é preciso uma investigação cuidadosa para que o paciente evolua com ausência de crises, de déficits neurológicos e possa melhorar sua qualidade de vida. Victor Horsley (apud Taylor) ${ }^{1}$ em 1887, descreveu a primeira cirurgia em paciente que apresentava crises parciais motoras. Ele encontrou uma lesão na área motora que foi ulteriormente diagnosticada como um tuberculoma e após a cirurgia as crises puderam ser controladas. Remoção de cicatrizes corticais, decorrentes de traumatismo cranioencefálico, que evoluíram com crises epilépticas, foi descrita por Foerster e Penfield ${ }^{2}$ em 1930, apresentando resultados positivos no controle das crises, mostrando uma nítida relação entre agressão cortical e crises epilépticas. Contudo, somente com o uso do eletrencefalograma (EEG) por Berger em 1929 (apud Engel) ${ }^{3}$ foi possível correlacionar alterações neurofisiológicas, observadas em traçados eletrencefalográficos, com lesões cerebrais. Esse exame teve grande contribuição no estudo de pacientes epilépticos, mostrando a localização das descargas paroxísticas focais, essencial em pacientes com epilepsia de difícil controle, pois, muitas vezes, não se encontram lesões estruturais em exames de neuroimagem.
\end{abstract}

Unitermos: Epilepsia, epilepsia de difícil controle, cirurgia das epilepsias.

Departamento de Neurologia e Neurocirurgia, Escola Paulista de Medicina - Universidade Federal de São Paulo.

* Professor-adjunto (Bolsista) da Disciplina de Neurocirurgia do Departamento de Neurologia e Neurocirurgia da Escola Paulista de Medicina - Unifesp.

** Médico Pós-graduando da Disciplina de Neurocirurgia do Departamento de Neurologia e Neurocirurgia da Escola Paulista de Medicina - Unifesp.

*** Médica Pós-graduanda da Disciplina de Neurocirurgia do Departamento de Neurologia e Neurocirurgia da Escola Paulista de Medicina - Unifesp.

**** Médico da Disciplina de Sono do Departamento de Psicobiologia da Escola Paulista de Medicina - Unifesp.

***** Médico do Setor de Neuropediatria da Disciplina de Neurologia do Departamento de Neurologia e Neurocirurgia da Escola Paulista de Medicina - Unifesp.

****** Médica do Setor de Neurofisiologia da Disciplina de Neurologia do Departamento de Neurologia e Neurocirurgia da Escola Paulista de Medicina - Unifesp.

******* Médica do Setor de Neuropediatria da Disciplina de Neurologia do Departamento de Neurologia e Neurocirurgia da Escola Paulista de Medicina - Unifesp.

******** Psicóloga Pós-graduanda do Setor de Investigação e Tratamento das Epilepsias da Disciplina de Neurologia do Departamento de Neurologia e Neurocirurgia da Escola Paulista de Medicina - Unifesp.

********* Assistente Social do Setor de Neuropediatria da Disciplina de Neurologia do Departamento de Neurologia e Neurocirurgia da Escola Paulista de Medicina - Unifesp. 


\section{Introdução}

Pacientes com epilepsia refratária ao tratamento clínico podem se beneficiar com a cirurgia para a remoção do foco cortical epileptiforme ou com a interrupção da propagação desse foco. $\mathrm{Na}$ indicação de uma cirurgia é necessária uma investigação muito cuidadosa para que o paciente evolua sem crises, sem déficits neurológicos e que melhore sua qualidade de vida. Victor Horsley (apud Taylor, D.C.) ${ }^{1}$ em 1887, descreveu a primeira cirurgia indicada em um paciente com crises parciais motoras. Encontrou uma lesão na área motora, que foi posteriormente diagnosticada como tuberculoma, e referiu que após a cirurgia houve controle das crises. A remoção de cicatrizes corticais, ocasionadas por trauma cranioencefálico, levando a crises epilépticas, foi referida por Foerster e Penfield ${ }^{2}$, em 1930, com ótimos resultados em relação ao controle das crises, mostrando uma nítida correlação entre a área cortical irritativa e as crises epilépticas. Entretanto, somente com a descrição clínica do uso da eletrencefalografia (EEG) por Berger, 1929 (apud Engel J.) ${ }^{3}$ que foi possível correlacionarmos as alterações neurofisiológicas observadas pelos traçados eletroencefalográficos com as lesões cerebrais. Exame este que trouxe grande contribuição no estudo dos pacientes epilépticos, pois demonstra a localização das descargas paroxísticas focais, o que é essencial nos pacientes com epilepsia de difícil controle, pois muitas vezes não encontramos lesões estruturais cerebrais nos exames de neuroimagem. O objetivo deste trabalho é fazer uma abordagem sobre as técnicas de investigação e as cirurgias das epilepsias fundamentadas na literatura analisada.

\section{Investigação}

$\mathrm{Na}$ investigação devem ser realizados exames para a localização da área epileptógena, como eletrencefalografia convencional ou com eletrodos especiais (nasofaríngeos, esfenoidais, zigomáticos). Em alguns casos, para uma localização mais exata do foco é necessário o uso de eletrodos epidurais, ou até corticais, que podem ser uados em série: strips colocados através de uma trepanação, ou placas grids colocadas por craniotomia; eletrodos de profundidade colocados por extereotaxia ou por neuronavegação (Olivier et al. ${ }^{4}$ ) (Figuras 1 e 2). A monitorização prolongada com vídeo/EEG, usando esses eletrodos, se faz nescessária. Outros exames podem fazer parte da investigação, como o SPECT cerebral, que é obtido pela emisssão de fóton único após a administração de tecnécio 99 endovenoso, sendo avaliado o fluxo cerebral por imagem. Assim, observamos, nos pacientes epilépticos, uma hipoconcentração focal no período interictal e uma hiperconcentração no período ictal. Ainda o SPECT cerebral é usado para a determinação de zonas eloqüentes cerebrais, que poderão ser confirmadas pela estimulação cortical, nos dando maior segurança para a remoção de áreas lesionais (Olivier et al. ${ }^{4}$ ) (Figura 3), com menor risco de seqüelas neurológicas. No PET cerebral a imagem é obtida por emissão de pósitron, sendo avaliado o metabolismo cerebral através da injeção de glicose endovenosa, no momento ictal a imagem interpretada é de aumento do metabolismo cerebral localizado, e no período interictal observamos uma diminuição desse metabolismo no local. A ressonância magnética encefálica poderá mostrar pequenas lesões expansivas epileptógenas ou áreas de escleroses mesiais temporais ou displasias corticais, que geralmente não são observadas pela tomografia computadorizada craniana. Esses exames deverão ser discutidos em reunião com a equipe médica especializada.

\section{Cirurgias do lobo temporal}

O tratamento cirúrgico dos pacientes com epilepsia do lobo temporal, de difícil controle, depende do tipo de crises e do achado em exames neurofisiólogicos, de neuroimagens e de testes neuropsicológicos. A primeira lobectomia temporal, realizada em pacientes com crises epilépticas de difícil controle foi descrita por Penfield (apud Penfield e Flanigin) ${ }^{5}$ em 1936 no Instituto Neurológico de Montreal, Canadá. Quando esta é indicada no hemisfério dominante, devemos respeitar o giro temporal superior na sua porção superior, o giro frontal inferior (fala motora), e o giro temporal posterior na sua porção superoposterior, giro supramarginal e angular e lobo parietal inferior (fala sensitiva), e ainda não deve ser lesada à veia de Labbe e porção lateroinferior do giro pré-central, sulco central e giro pós-central. Na porção inferior, na ressecção do hipocampo, devemos ter cuidado com o mesencéfalo e com as estruturas vasculares dessa região. $\mathrm{Na}$ ressecção parcial (ou polar) do lobo temporal, a cirurgia trará menos riscos de seqüelas ao paciente. Outra contribuição importante para a cirurgia da epilepsia foi a amídalohipocampectomia seletiva por via transcortical, transventricular, descrita por Niemeyer ${ }^{6}$, em 1958. Essa técnica ainda hoje é realizada pela facilidade de abordar a região hipocampal, sem a necessidade de manipular estruturas vasculares importantes do vale sylviano como a via descrita por Weiser \& Yasargil ${ }^{7}$, 


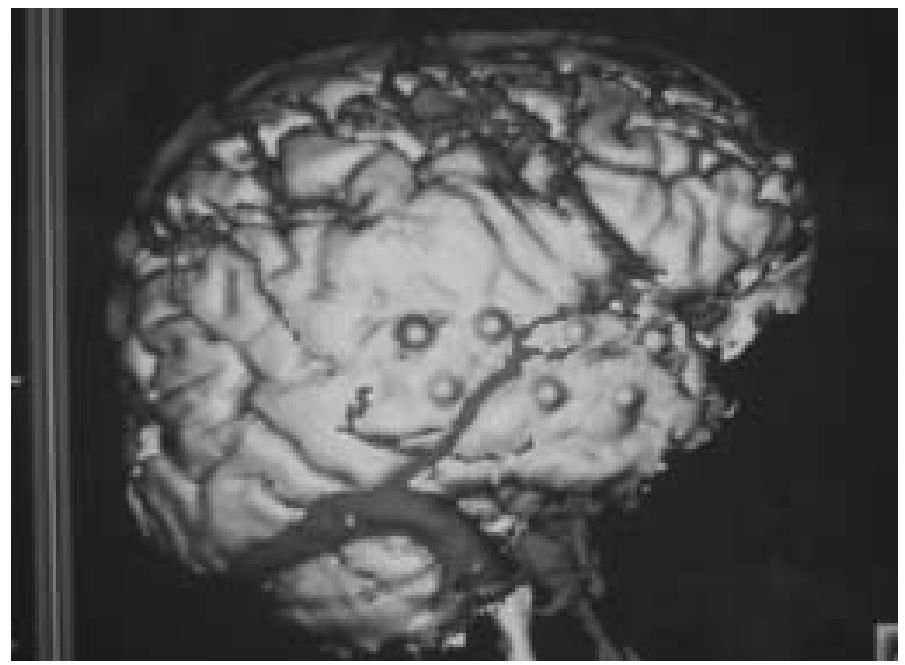

Figura 1 Podemos observar a marcação prévia da colocação de eletrodos de profundidade na região temporal por neuronavegação (Oliver et al. Instituto Neurológico Montreal).

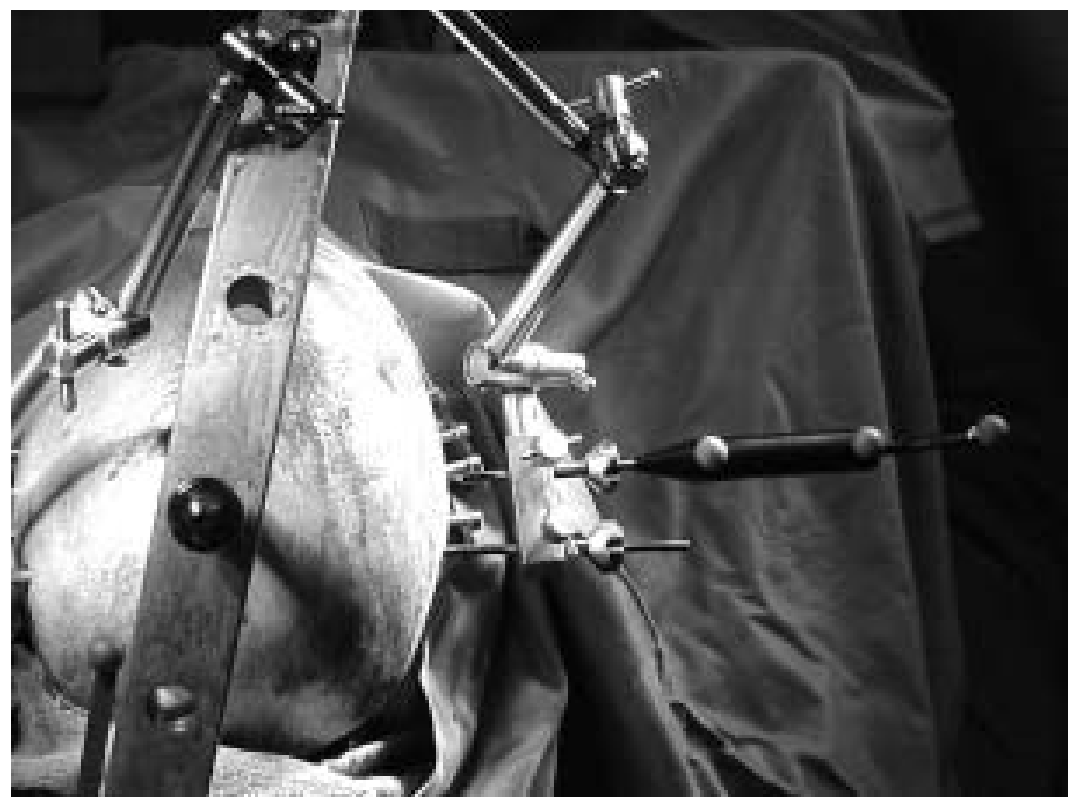

Figura 2 O fixador de cabeça vertical permite maior liberdade técnica para a colocação dos eletrodos temporais de profundidade. Podemos observar o pointer do neuronavegador (à direita da figura, com três esferas para a reprodução da localização no monitor) apontando para o hipocampo (Olivier et al., Instituto Neurológico de Montreal)4.

em 1982. Falconer e Calvanagh ${ }^{8}$ (1959) submeteram 23 pacientes à lobectomia total, por epilepsia de difícil controle, e o exame histopatológico mostrou tratarse de neoplasias em 13 deles, portanto as neoplasias devem ser sempre consideradas em pacientes que apresentam crises epilépticas. Rassi-Neto et al. ${ }^{9}$ apresentaram uma casuística de 32 pacientes com epilepsia temporal, refratárias ao tratamento medicamentoso. O EEG mostrava descargas paroxísticas focais na região temporal em todos os casos. Os casos foram dividido em dois grupos: 1) Composto de 18 pacientes cujos exames por neuroimagem (ressonância magnética e tomografia computadorizada craniana) mostraram imagens compatíveis com lesões expansivas cerebrais (gliomas, malformações arteriovenosas, tumor epidermóide, etc.). Foi realizada a exérese da lesão em todos os casos e associada à área cortical irritativa adjacente, identificada pela eletrocorticografia $(E C o G)$ intraoperatória em seis casos. Só foi possível a exérese dessas áreas irritativas nos casos em que essas localizavam-se em zonas não eloqüentes. 2) Composto de 14 pacientes, nos quais a ressonância magnética não mostrou imagem compatível com o processo expansivo cerebral, sendo 


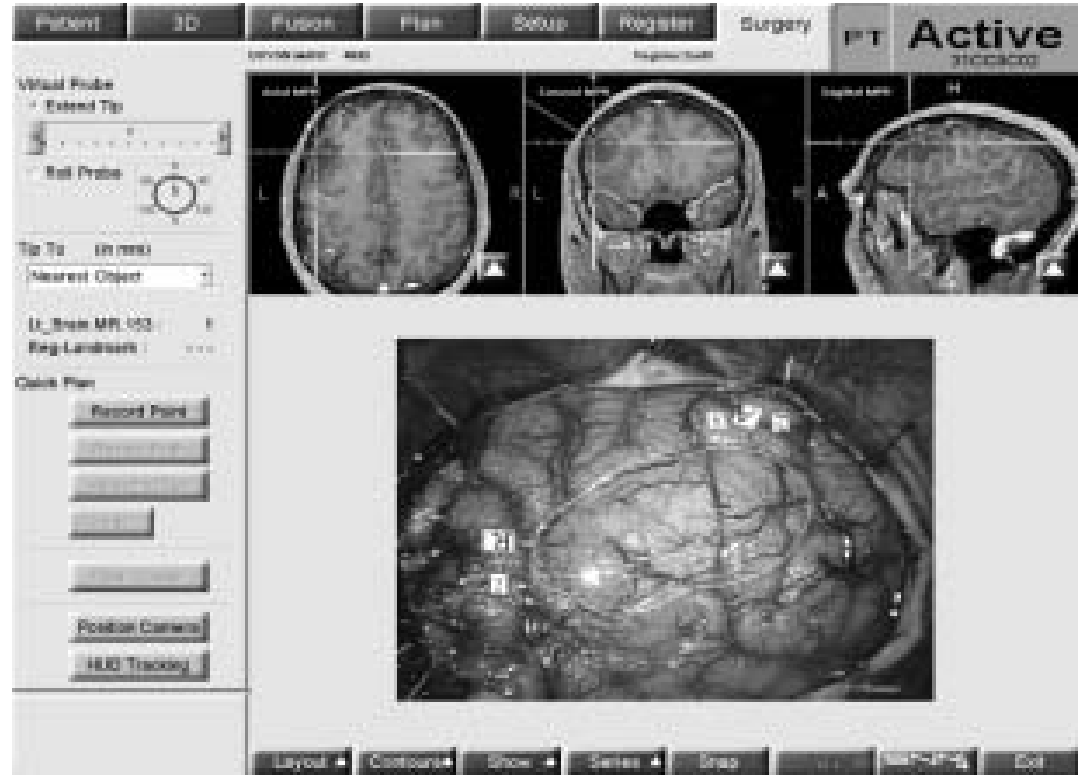

Figura 3 Na tela do monitor do neuronavegador pode ser observado, na parte superior, três imagens de ressonância magnética sobrepostas à imagem do SPECT cerebral. A área da lesão cerebral está demarcada pelo círculo central maior e na imagem da ressonância do centro, aparece uma linha proveniente do neuronavegador apontando para o centro da lesão, e os círculos superiores e inferiores à lesão demonstram a área da fala determinada pelo SPECT. Na parte inferior da figura observamos a foto cirúrgica com a marcação da lesão (linha central), e números e letras mostrando a área da fala confirmada pela estimulação cortical (Olivier et al., Instituto Neurológico de Montreal $)^{4}$.

submetidos à lobectomia temporal parcial. Foi observado que os casos com lesões expansivas cerebrais apresentaram melhor evolução em relação ao controle das crises epilépticas, do que os casos sem essas lesões, pois $15(83,4 \%)$ dos 18 casos do grupo 1 evoluiram sem crises e $10(71,4 \%)$ dos 14 casos do grupo 2 também evoluíram sem crises após a cirurgia.

A nossa casuística atual, com seguimento mínimo de 12 meses em pacientes com epilepsia do lobo temporal, consta de 108 pacientes submetidos à ressecção parcial temporal e/ou lesionectomia. A lesão cerebral estrutural mais freqüente foi a esclerose mesial temporal, seguida da displasia cerebral. Em 38 pacientes foram observadas pequenas lesões expansivas epilepitógenas: astrocitomas (15 casos), oligodendrogliomas ( 2 casos), malformações arteriovenosas ( 2 casos), cavernomas ( 9 casos), neurocisticercose (3 casos), processo inflamatório crônico ( 1 caso), cisto de aracnóide ( 1 caso), tumor epidermóide ( 2 casos), granuloma enespecífico (1 caso), gliose (1 caso), meningioma angioblástico (1 caso). Para descrevermos a evolução de nossa casuística, em relação à presença das crises epilépticas, tomamos por base a classificação de Engel (1989) ${ }^{10}$, UCLA: I) pacientes que evoluíram sem crises ou com apenas algumas crises no pós-operatório imediato e após houve controle completo; II) evidente melhora na freqüência das crises (> 80\%); III) inalterado em relação à freqüência das crises. Para os 38 (dentre os 108) pacientes submetidos à lesionectomia, observamos a seguinte evolução na classificação de Engel: na I em $88 \%$, na II em 95\%. Nos demais, 70 pacientes, submetidos à lobectomia temporal parcial (com hipocampectomia) observamos uma evolução na classificação I em $72 \%$, na II em $90 \%$.

\section{Cirurgias extratemporais}

Algumas síndromes epilépticas ocorrem na infância, tais como as crises neonatais, espasmos infantis, a síndrome de Lennox-Gastaud, SturgeWeber, pequeno mal e outras. O substrato patológico dessas crises são muito diferentes daqueles que causam crises no adulto. Assim, acredita-se que devemos seguir um protocolo cirúrgico específico para crianças com epilepsia de difícil controle (Shields et al. $)^{11}$.

Existem muitos fatores importantes na maturação e no desenvolvimento do cérebro, tais como a arborização dendrítica, a sinaptogênese, a mielinização e a proliferação das células gliais. Pela repetitiva proliferação de ramificações dendríticas forma-se uma enorme superfície que serve como sítios receptores para o estabelecimento de sinapses. A maturação cortical relaciona-se bem com o grau de ramificações 
dendríticas e o número de sinapses, cuja densidade máxima dá-se aos oito anos de vida decrescendo para um plateau aproximadamente aos 14 anos de idade (Huttenlocher) $^{12}$.

É difícil responder se as crises interferem com esse processo de maturação. Entretanto, é conhecido que a sinaptogênese adequada é dependente de um campo elétrico normal, e sob condições experimentais, onde foram induzidas descargas epileptiformes, o crescimento axonal sofreu interferência do seu curso normal (Kellaway) ${ }^{13}$. A depressão pós ictal, que é possívelmente devido à liberação de opióides endógenos, provavelmente também interfere com a sinaptogênese normal (Engel et al. $)^{14}$. Pontas interictais causam alterações na citoarquitetura em animais experimentais (Crowell, Ebersole et al.) $)^{15,16}$. Drogas antiepilépticas também podem interferir na maturação cortical (Diaz et al., Diaz et al.) ${ }^{17,18}$. Sendo assim, há muitas evidências para a idéia de que crises freqüentes afetam de maneira adversa a maturação do cérebro humano. Isso sugere, então, que se crises incontroláveis ocorrem cedo, na infância, e uma lesão possível de ser removida pode ser encontrada, o período mais apropriado para intervenção cirúrgica é o mais cedo possível. Com as crises eliminadas, a maturação poderá processar-se sem interferências (Duchowny et al. $)^{19}$.

\section{Ressecção frontal}

$\mathrm{Na}$ lobectomia frontal devemos estar atentos para as estruturas vasculares e nervos da porção inferior do lobo frontal e na porção posterior do lobo frontal (giro pré-central, sulco central e giro pós-central) que corresponde à área motora, e na sua porção lateroinferior (giro frontal inferior) que corresponde à área da fala motora, no hemisfério dominante. A identificação do giro pré e pós-central é melhor estabelecida pela estimulação cortical sob anestesia local. A ressecção é feita em bloco, dos três giros frontais horizontais, sobre a convexidade lateral e é seguida pela ressecção do giro anterior do cíngulo ao redor do corpo caloso. No lado dominante, a ressecção estende-se até a metade do segundo giro frontal ou, de preferência, até o sulco frontal inferior. Os $2,5 \mathrm{~cm}$ posteriores do giro frontal inferior são deixados intactos para evitar distúrbios da fala. Nessa região costuma-se dar mais importância para os parâmetros anatômicos e topográficos do que respostas à estimulação. Ainda no lobo frontal, em alguns casos estão indicadas ressecções mais localizadas como as paramedianas, frontopolares e de convexidade.

\section{Ressecção central}

A grande maioria dos pacientes com crises epilépticas na região central apresentam crises parciais motoras e/ou somatossensoriais. As crises permanecem localizadas em alguns pacientes, mas em outros elas progridem para generalização secundária. Status epilepticus com crises parciais contínuas são particularmente comuns (Rasmussen) ${ }^{20}$. Ressecção da área central inferior, incluindo os giros pré e pós-central deverá ser feita mesmo no hemisfério dominante. A ressecção deve estender-se a uma distância próxima de $3 \mathrm{~cm}$ acima da fissura silviana para evitar déficit nas áreas da língua, do polegar ou lábios, que deverão ser bem identificadas por estimulação cortical sob anestesia local.

\section{Ressecção parietal}

Ressecções no lobo parietal são possíveis somente após meticulosa identificação do giro pós-central. Tanto quanto na região central, é de máxima importância respeitar e deixar intactas quaisquer veias ascendentes para o seio sagital superior responsáveis pela drenagem venosa do lóbulo paracentral. Indicações de cirurgia dessa região são baseadas numa forte congruência de dados clínicos, neuroimagem e eletrencefalográficos. Registros intracranianos podem ser necessários para estabelecer a participação da área central nas descargas epilépticas.

\section{Ressecção occiptal}

As crises epilépticas do lobo occipital manifestam-se por sintomas visuais, tais como cegueira ou alucinações visuais transitórias caracterizadas por flash de luz, bolas coloridas e outros padrões geométricos que nunca são completamente organizados. A cirurgia no lobo occipital só é realizada se houver uma forte evidência do foco epileptiforme, usualmente comprovado por eletrodos intracerebrais. São raras as indicações da ressecção occipital, assim como da parietal, e quase sempre são seguidas de hemianopsia. As ressecções multilobares devem ser indicadas em alguns casos.

\section{Transecções subpiais múltiplas}

Introduzida em 1989 por Frank Morrell et al. ${ }^{21}$, a transecção subpial múltipla (TSM) foi idealizada para os pacientes com epilepsia de difícil controle com descargas epileptiformes localizadas nas áreas eloqüientes. 
Estudos neurofisiológicos e anatômicos em animais demonstraram que os focos epileptiformes, separados por 6-7 $\mathrm{mm}$, descarregavam independentes, mas se eles estivessem menos que $4 \mathrm{~mm}$ um do outro, descarregavam sincronicamente (Luders et $a l.)^{22}$. Muitos estudos têm demonstrado a importância das propagações para áreas adjacentes ao foco epiléptico (Szentagothai) ${ }^{23}$. Morrell et al. ${ }^{21}$ propuseram que se a organização colunar fosse deixada intacta e a propagação horizontal para regiões adjacentes fosse interrompida por transecções subpiais separadas por $5 \mathrm{~mm}$, então a massa crítica dos neurônios com descargas sincrônicas poderia cessar, mantendo-se intacta a coluna vertical, preservando, portanto, a função do córtex operado. São realizadas, então, transecções subpiais, aproximadamente $5 \mathrm{~mm}$ de distância uma da outra, perpendiculares ao eixo longo do giro, naqueles casos em que a área epileptógena localiza-se nas regiões corticais eloqüentes, que se removidas acarretaria danos neurológicos ao paciente.

\section{Hemisferectomia}

A hemisferectomia está indicada em casos com zonas irritativas corticais amplas, como na síndrome de Rasmussen, Sturge-Weber, e em outras lesões estruturais hemisféricas associadas a atividades epileptiformes. A síndrome de Rasmussen é descrita como uma encefalite progressiva causando hemiparesia, retardo mental e epilepsia de difícil controle. Os exames por neuroimagem, especialmente a ressonância magnética, mostram imagens sugestivas de atrofia cortical e gliose. O exame histopatológico mostra perda neuronal e infiltrado linfocitário perivascular. Kenneth McKenzie em 1938 (apud Shields et al. $)^{11}$ foi o primeiro cirurgião a realizar uma hemisferectomia em um paciente com epilepsia de difícil controle. Em 1950, Roland Krynauw (apud Shields et al. $)^{11}$ descreveu a realização desse procedimento em 12 crianças com epilepsia intratável e todas apresentavam hemiparesia anterior à cirurgia.

Entretanto, a hemisferectomia anatômica, por ser um procedimento muito amplo, trouxe também complicações tardias no pós-operatório, incluindo alta mortalidade. Em 1983, Rasmussen ${ }^{24}$ descreveu a hemisferectomia funcional, que constitui a lobectomia temporal (incluindo hipocampo e amígdala), remoção dos giros pré e pós-centrais com desconecção do lobo frontal e parietal, sem removê-los totalmente, e calosotomia. Modificações das hemisferectomias funcionais (Schramm et al. $)^{25}$ têm sido propostas para reduzir a remoção cerebral conseqüentemente à incidência de hemosiderose, diminuindo a morbidade e mortalidade, mantendo a mesma eficácia no controle das crises.

\section{Calosotomia}

A divisão cirúrgica do corpo caloso para acesso ao terceiro ventrículo, como descrito por Dandy em $1931^{26}$, foi utilizada, posteriormente, para a cirurgia da epilepsia. A idéia desse procedimento para cirurgia de epilepsia emerge da observação feita por Van Wagenen and Herren (Van Wagenen et al.) ${ }^{27}$ sobre pacientes epilépticos que após apresentarem tumores ou infartos envolvendo o corpo caloso melhoraram suas crises. Os benefícios desse procedimento a longo prazo, na melhora da frequência das crises, não foi devidamente apreciado naquela época (Bogen et al., Luessenhop et al. $)^{28,29}$ devido ao pequeno número de casos e ao follow up curto dessas séries. A série de Dartmouth (Harbaugh et al., Reeves et al., Roberts, Wilson et al., Wilson et al. $)^{30,31,32,33,34}$ representou um programa envolvendo mais de 100 pacientes, sendo que o sucesso encontrado nessa série, junto com outras experiências similares em outras partes do mundo, consolidou a utilidade dessa técnica no tratamento cirúrgico da epilepsia. Ao contrário das cirurgias ressectivas (lobectomias, hemisferectomias, ressecções corticais) a calosotomia é uma cirurgia de desconexão, que limita a propagação das descargas, geralmente bilaterais, de um hemisfério para o outro. Aqueles pacientes cujas crises são caracterizadas por drop attacks (tônicas, atônicas) têm, geralmente, os melhores resultados após a secção do corpo caloso. Os resultados geralmente não são satisfatórios para os pacientes com outros tipos de crise.

A calosotomia é realizada por via de acesso frontal, inter-hemisférica, e sob microscopia cirúrgica realizamos a secção dos $2 / 3$ anteriores do corpo caloso, pois se a lesão cirúrgica for mais extensa poderá ocorrer síndrome de desconexão, entretanto, alguns autores indicam a secção total, esperando um melhor controle das crises. A indicação desse procedimento está cada vez mais restrito, pois a hemisferectomia, quando possível sua indicação, tem demonstrado resultados mais animadores (apud Olivier e Awad) ${ }^{35}$.

\section{Comentários}

Segundo a classificação de Engel ${ }^{10}$ a lobectomia temporal apresenta uma evolução sem crises (classe I), em torno de $55,5 \%$, e uma redução > $90 \%$ das crises (classe II) em torno de $27,7 \%$, observado após 
a realização de 2.336 lobectomias. Na amidalohipocampectomia seletiva Yasargil et al., 1993 ${ }^{36}$, observaram uma evolução class I em $57 \%$ e classe II em $9 \%$ de 100 casos operados. Rassi Neto et al., ${ }^{9}$, estudaram 32 pacientes com epilepsia, difícil controle associada a lesões temporais, sendo um grupo com esclerose hipocampal e outro grupo com pequenas lesões expansivas. Todos os pacientes foram submetidos à cirurgia, no primeiro grupo mencionado houve uma evolução sem crises em 71,4\% dos casos e no segundo grupo em $83,4 \%$. Do total (32 casos) observaram uma evolução sem crises em $78,1 \%$ dos casos.

Nas ressecções corticais (extratemporal) Engel ${ }^{10}$, encontraram $42,3 \%$ classe I e $27,8 \%$ classe II, de 825 casos operados. A hemisferectomia mostrou classe I em $77,3 \%$ e classe II em $18,2 \%$. Na calosotomia, encontrou 5\% classe I e $71 \%$ classe II, de 197 casos operados. Na Conferência de Palm Desert (EUA) sobre epilepsia, em 1992, 91 centros relataram 5.746 cirurgias e seus resultados no período de 1986 a 1990 (Engel et al. $)^{10}$. Nessa casuística numerosa, $66 \%$ das cirurgias eram temporais, $18 \%$ extratemporais, $10 \%$ calosotomias e $6 \%$ hemisferectomias.

Os resultados das ressecções extratemporais geralmente têm sido substancialmente piores do que as cirurgias do lobo temporal, particularmente na ausência de lesões. Existe uma série de razões para isso: 1) Aparentemente há mais zonas epileptogênicas difusas na epilepsia extratemporal. 2) Uma difusão mais rápida das crises dificultando a identificação eletrofisiológica dessas zonas. 3) Maior freqüência de sobreposição com áreas eloqüentes impondo limitações na ressecção completa da região epileptógena. A hemisferectomia é um procedimento cirúrgico com excelentes resultados no controle das crises, isto é, de $75 \%$ a $85 \%$ dos casos (Engel; Davies et al.) ) $^{3,37}$ apresentam uma evolução sem crises e com índices cada vez menores de complicações devido às novas técnicas de hemisferectomia funcional.

Analisando a casuística da Dartmouth Medical School, Roberts ${ }^{32}$ e Wilson et al. ${ }^{34}$ observa-se que $43 \%$ dos pacientes submetidos à calosotomia devido à drop attacks evoluíram sem crises e uma redução de no mínimo $80 \%$ na freqüência dessas crises.

Existem vários procedimentos cirúrgicos para o tratamento da epilepsia de díficil controle, entretanto, acreditamos que o uso da eletrocorticografia intraoperatória para a confirmação da localização exata da área irritativa, a estimulação cortical, para evitarmos lesões de áreas eloqüentes, instrumentos intra-operatórios como a extereotaxia e o ultra-som para localização de pequenas lesões epileptógenas, como cavernomas subcorticais, neurocisticercose ou tumores de baixo grau (Falconer e Calvanagh, Spencer et al., Boon et al., Kirkpatrick et al., Pilcher et al., Rassi Neto et al., Rassi-Neto et al., Oliveira et al., Rassi Neto et al., Rassi Neto et al., $)^{8,38,28,39,40,9,41,42,43,44}$ devem ser usados, pois o objetivo dessas cirurgias é reduzir ao máximo a frequiência das crises sem deixar déficits neurológicos nos pacientes. O acompanhamento ambulatorial pós-operatório deve ser realizado pela mesma equipe médica e paramédica, que apoiará o paciente para a nova fase de sua vida, sem crises epilépticas na maioria das vezes, surgindo uma melhor qualidade de vida.

\section{SUMMARY}

\section{Surgical treatment of epilepsy}

Patients with difficult-control epilepsy may have the benefit of a surgery to remove the epileptiform cortical focus or to interrupt these foci. To perform the surgery, a careful investigation is needed in order that the patient may evolve without seizures, neurological deficits and that he/she could improve his/her quality of life. Victor Horsley (apud Taylor) ${ }^{1}$, in 1887, described the first surgery in a patient presenting partial motor seizures. He found a lesion in the motor area, which was later diagnosed as a tuberculoma and after surgery the seizures could be controlled. Removal of the cortical scars due to cranioencephalic trauma leading to epileptic seizures, was also mentioned by Foerster e Penfield ${ }^{2}$ in 1930, presenting successful results to control seizures, showing a clearly correlation between the irritative cortical area and epileptic seizures. However, only after the clinical description of the electroencephalography (EEG) by Berger, 1929 (apud Engel) ${ }^{3}$ that it was possible to correlate neurophysiological alterations observed by electroencephalographic traits with cerebral lesions. This test much contributed to the study of epileptic patients, showing the location of the focal paroxysmal discharges which is essential in patients with a difficult-control epilepsy, since many times we are not able to find evident cerebral structural lesions in neuroimage investigation.

\section{Keyw ords}

Epilepsy, difficult-control epilepsy, epilepsy surgery. 


\section{Referências}

1. Taylor DC. One hundred years of epilepsy: Sir Vitor Horsley contribuition. In: Engel J. (ed.) Surgical treatment of epilepsies. New York, Raven Press, 1987, pp. 7-11.

2. Foerster $\mathrm{O}$ e Penfield $\mathrm{W}$. The structural basis of traumatic epilepsy and results of radical operation. Brain, 53:8119, 1930.

3. Engel Jr J. Epilepsy then historical perspective. In: Engel Jr. J (Ed). Seizures and epilepsy. Philadelphia, F.A. Davis Company, 1989, pp. 22-37.

4. Olivier A, Alonso-Vanegas M, Comeau R, Peters TM. Image-guided surgery of epilepsy. Neurosurg Clinics of North America, 7(2):229-43, 1996.

5. Penfield W, e Flanigin H. Surgical therapy of temporal lobe seizures. Arch Neurol Psychiatry, 64:491-500, 1950.

6. Niemeyer $P$. The transventricular amydalo hipocampectomy in temporal lobe epilepsy. In: Baldwin M, Bailey $P$ (ed.). Temporal lobe epilepsy. Illinois, Thomas Springfield, 1958. pp. 461-82.

7. Wieser HG, Yasargil MG. Selective amydalo hippocampectomy as a surgical treatment of mesiobasal limbic epilepsy. Surg Neurol, 17:445-57, 1982.

8. Falconer MA, Cavanagh JB. Clinico-pathological considerations of temporal lobe epilepsy due to small focal lesions. A study of cases submitted to operation. Brain, 82(4):484-504, 1959.

9. Rassi Neto A, Campos CJR, Muskat M, Ferraz FP. Epilepsia do lobo temporal: tratamento cirúrgico. Arq Neuro-Psiquiatria, 54(4): 618-27, 1996.

10. Engel J Jr, Van Ness P, Rasmussen $\mathrm{T}$ et al. Outcome with respect to epileptic seizures, $2^{\text {nd }}$ In: Engel Jr J (ed). Surgical treatment of epilepsies. New York, Raven Press, 1993.

11. Shields WD, Peacock WJ, Roper SN. Surgery for epilepsy: Special pediatric considerations. Neurosurgery Clinics of North America. In: Silbergeld DL, Ojemanjn G (Eds.), Philadelphia, W.A. Company, 1993, pp. 301-10.

12. Huttenlocher PR. Dendritic development in neocortex of children with mental defect and infantile spasms. Neurology, 24:203-210, 1974.

13. Kellaway P. Afferent input: a critical factor in ontogenesis of brain electrical activity. In: Burch N, Altshuber HL (eds.). Behavior and brain electrical activity. New York, Plenum Publishing, 1975 pp. 391-420.

14. Engel Jr J, Wilson CL. Evidence of enhanced synaptic inhibition in human epilepsy. In: Morselli PL, Nistico G, Engel Jr J, Farello RG, Lloyde KG (Eds.). Neurotransmitters, Seizures and Epilepsy III, New York, Raven Press, 1986, pp 1-10.

15. Crowell RM. Distant effects of a focal epileptogenic process. Brain Res, 18:137-54, 1970.

16. Ebersole JS, Levine RA. Abnormal neuronal responses during evolution of a penicillin epileptic focus in cat visual cortex. J Neurophysiol, 38:250-66, 1975.

17. Diaz J, Schain RJ. Phenobarbital: effects of long term administration on behavior and brain of artificially reared rats. Science, 199:90-1, 1978.

18. Diaz J, Shields WD. Effects of dipropylacetato on brain development. Ann Neurol, 10:465-8, 1981.

19. Duchowny M, Jayakar P, Resnick T, Harvey AS, Alvarez L, Dean P, Gilman J, Yaylali, Morrison G, Prats A, Altman $\mathrm{N}$, Birchansky S, Bruce J. Epilepsy surgery in the first three years of life. Epilepsia, 39(7):737-43, 1988.
20. Rasmussen T. Surgery of frontal lobe epilepsy. Adv Neurol, 8:139-44,1975.

21. Morrell F, Whisler WW, Bleck TP. Multiple subpial transection: new approach to the surgical treatment of focal epilepsy. J Neurosurg, 70:231-9,1989.

22. Luders H, Bustamenete L, Zablow L et al. Quantitativa studies of spike foci induced by minimal concentrations of penicillin. Eletroencephalogr Clin Neurophysiol, 48:809,1980 .

23. Szentagothai J. The use of degeneration methods in the investigation of short neuronal connections. Prog Brain Res, 14:1-32, 1965.

24. Rasmussen T. Surgical treatment of complex parcial seizures: results, lessons and problems. Epilepsia, 24(suppl 1):65-76,1983.

25. Schramm J, Behrens E, Entzian W. Hemispherical deafferentation: an alternative to funcional hemispherectomy. Neurosurgery, 36:509-18, 1995.

26. Dandy WE. Congenital cerebral cists of the cavum septi pellucidi (fifth ventricle) and cvum vergae (sixth ventricle): Diagnosis and treatment. Archives of Neurology and Psychiatry, 25:44-66,1931.

27. Van Wagenen WP, Herren RY. Surgical division of the commissural pathways in the corpus callosum: ralation to spread of na epileptic attack. Applied Neurophisyology, 44: 740-59, 1940.

28. Bogen JE, Fisher ED, Vogel PJ. Cerebral commissurotomy: A second case report. JAMA, 194:1328-9,1965.

29. Luessenhop AJ, Dela-Cruz TC, Fenichel GM. surgical desconection of the cerebral hemispheres for intractable seizures. JAMA, 213:1630-6, 1970.

30. Harbaugh RE, Wilson DH, Reeves AG et al. Forebrain commissurotomy for epilepsy: review of 20 consecutive cases. Acta Neurochir (Wien), 68:263-75, 1983.

31. Reeves AG, O'leary PM. Total corpus callosotomy for control of intractable epilepsy. In: Reeves AG (ed.). Epilepsy and the Corpus Callosum. New York, Plenum Press, 1985, pp. 269-80.

32. Roberts DW. Corpus callosotomy: surgical technique In: Reeves AG (ed.). Epilepsy and the Corpus Callosum. New York, Plenum Press, 1985, pp. 259-67.

33. Wilson DH, Reeves AG, Gazzaniga M et al. Cerebral commissurotmy for control of intractable seizures. Neurology, 27:708-15,1977.

34. Wilson DH, Reeves AG, Gazzaniga M. "Central" commissurotomy for intractable generalized epilepsy: series two. Neurology, 32:687-97,1982.

35. Olivier A, Awad I. Extratemporal resections. Surgical treatment of epilepsies. In: Engel Jr J (ed.). $2^{\text {nd }}$, New York, Raven Press, 1993, pp. 489-500.

36. Yasargil MG, Weiser HG, Valavanis A, Ammon K, Roth P. Surgery and results of selective amygdala-hippocampectomy in one hundred patients wiyh nonlesional limbic epilepsy. Neurosurgery Clinics of North America, 4:24361, 1993.

37. Davies K, Maxwell R, French L. Hemispherectomy for intractable seizures: long-term results in 17 pacients, followed by up to 38 years. J Neurosurg, 78:733-39, 1993.

38. Spencer DD, Spencer SS, Mattson RH, Williamson PD. Intracerebral masses in patients with intractable partial epilepsy. Neurology, 34:432-6, 1984.

39. Kirkpatrick PJ, Honavar M, Janota I, Polkey CE. Control of temporal lobe epilepsy following en bloc resection of low-grade tumors. J Neurosurg, 78:19-25, 1993. 
40. Pilcher WH, Silbergeld DL, Berger, MS, Ojemann GA. Intraoperative electrocorticography during tumor resection: impact on seizure outcome in patients with gangliogliomas. J Neurosurg, 78:891-902, 1993.

41. Rassi Neto A, Ribeiro PRJ, Prates MA, Muskat M, Campos CJR, Ferraz FAP. Tratamento cirúrgico das patologias vasculares cerebrais nos pacientes epilépticos. Arq Neuro-Psiquiatria, 55(3-A):408-12, 1997.

42. Oliveira CA, Rassi Neto A, Ferraz F. Meningioma angioblástico do hipocampo. Relato de caso. Jornal Brasileiro de Neurocirurgia, 8(2): 82-4, 1997.

43. Rassi Neto A, Centeno RS, Ferraz F. Tratamento cirúrgico da epilepsia associado à neurocisticercose. Jornal Brasileiro de Neurocirurgia, 9(3):99-102, 1998.
44. Rassi Neto A, Ferraz FP, Campos CJR, Braga FM. Patients with epileptic seizures and cerebral lesions who submitted to lesionectomy restricted or associated to the irritative area adjacent. Epilepsia, 40(7): 856-64,1999.

\section{Endereço para correspondência:}

Aziz Rassi Neto

Escola Paulista de Medicina - Universidade Federal de São Paulo

Disciplina de Neurocirurgia

Rua Botucatu, 740 - Vila Clementino

CEP 04023-900 - São Paulo, SP 